This is a self-archived version of an original article. This version may differ from the original in pagination and typographic details.

Author(s): Ostovar, Arash; Chang, Zheng

Title: Reducing power consumption of wireless networks through collaborative DMC mobile clusters

Year: 2018

Version: Accepted version (Final draft)

Copyright: @ Springer Science+Business Media, LLC 2017.

Rights: In Copyright

Rights url: http://rightsstatements.org/page/lnC/1.0/?language=en

Please cite the original version:

Ostovar, A., \& Chang, Z. (2018). Reducing power consumption of wireless networks through collaborative DMC mobile clusters. Wireless Personal Communications, 98(2), 1771-1784. https://doi.org/10.1007/s11277-017-4944-2 


\title{
Reducing Power Consumption of Wireless Networks through Collaborative DMC Mobile Clusters
}

\author{
Arash Ostovar · Zheng Chang
}

Received: date / Accepted: date

\begin{abstract}
Reducing the energy consumption of the wireless network is significantly important to the economic and ecological sustainability of the ICT industry, as high energy consumption may limit the performance of wireless networks and is one of the main network costs. To solve energy consumption problem, especially on the terminal side, a scheme known as distributed mobile cloud (DMC) is considered to be a potential solution. Multiple mobile terminals (MTs) can cooperative to take advantage of good quality links among the MTs to save energy when receiving from the Base Station (BS). In this paper, we aim to find the optimal transmit power to further reduce the energy consumption of DMC. From simulation studies, it is shown that up to $80 \%$ energy savings can be accomplished when using optimal transmit power, compared to use the standard DMC without exploring the optimal transmit power.
\end{abstract}

Keywords DMC $\cdot$ transmit power $\cdot$ power consumption · energy saving $\cdot$ green communications

\section{Background and Motivation}

The next generation of wireless networks are expect to provide fast Internet access to anyone at anytime at anywhere. Meanwhile, the exponentially developing information and communications technology (ICT) industry and the necessity of universal access have activated a sensational extension of system frameworks and quick acceleration of energy consumption. Subsequently, it turns into a requirement for portable administrators and operators to keep up feasible limit development and the power bill. It has been found that the overall energy used of $3 \%$ is devoured by ICT base

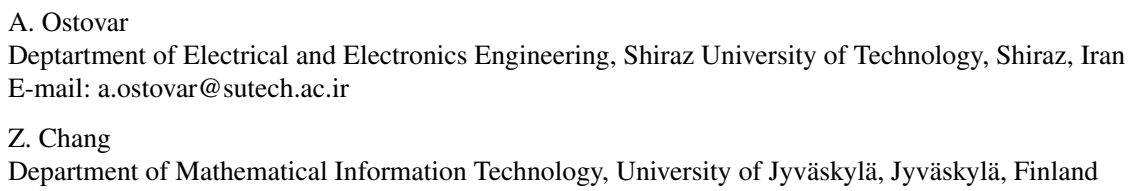


that causes around $2 \%$ of the overall carbon dioxide $\left(\mathrm{CO}_{2}\right)$ outflows [1]. The acceleration of energy consumption in wireless networks, brings about the expansion of nursery gas discharge, which has been perceived as a significant risk for natural insurance and supportable advancement [2]. The European Union has gone about as a main leader in energy saving in the world and focused to reduce a $20 \%$ nursery gas usage. To meet the difficulties raised by the appeal of wireless movement and energy consumption, green communications development, including the design of energyefficient communication infrastructures, protocols, devices, and services, has turned into an earnest requirement for wireless networks today. Hence, increasing the energy effectiveness of radio systems, in general, is a emergingly significant research area.

On the other hand, conventional studies on energy-constrained networks or green communications usually focused on designing the energy saving scheme proposals at the network or Base Station (BS) side. However, in many cases, the wireless networks performance is constrained by the other the network elements, such as mobile phones equipped with batteries. During the last decades, the battery capacity has improved at relatively slow speed which creates the bottleneck in prolonging the lifetime of the networks. Meanwhile, the rise of online services is drawing down the battery voltage of mobile devices much faster than before. All these issues have resulted in an interesting research topic about energy-efficient design of mobile terminals (MTs). In this paper, we are also aiming at proposing novel approaches to reduce the network energy cost, particularly from the terminal aspect.

\section{Related work}

The interest for energy-efficient networking has started to spread in the past decades and is continuing to increase. Besides the widespread sensitivity to natural issues, such intriguement originates from economic needs subsequent to both energy costs and electrical necessities of telecom operators infrastructures around the world demonstrate a constantly developing pattern. Since the fact that the BSs are not being used in the most optimal and efficient way, a considerable measure of cash in the mobile networks is being wasted, especially in the BS sides [3]. In addition, operating costs are being lifted as a result of the carbon footprint expansion of the wireless networks. This expansion is because of the way that the mobile networks are developing more and more repidly together with the data volumes required by the growing number of network users [3]. The energy consumption of a typical mobile phone operator's radio base station (RBS) site makes the most part out of the power use of the RBS itself, mobile telephone exchange (MTX), and the core network. Data centers and partial energy consumption, which have more of a supportive role in the big picture, play quite an insignificant role [4]. From the energy consumption perspective, the BS includes power supply, radio frequency (RF) transceiver, climate control, and battery backup, as well as an optional transmission link in the baseband unit in the bigger BSs to connect to the operator's network for platform control and medium access control. The RBS itself is comprised of the power amplifier (PA) and multiple transceiver units. At full load the BS's radio units command the site's power con- 
sumption, which is completely sensible; however at almost zero burdens regardless, they stay noteworthy [4].

Recently, there are considerable number of research work dedicated to find novel energy saving approaches such as, mobile offloading, cloud-RAN etc. In [5], the authors investigated the mobile edge computing offloading mechanisms in $5 \mathrm{G}$ heterogeneous networks. To solve the problem of energy consumption minimization, they proposed an energy-efficient computation offloading scheme, which jointly optimized the calculation offloading decisions and the radio resource allocation techniques to minimize the system energy cost under the delay limitations. The authors of [6] analyzed the energy efficiency performance optimization for heterogeneous cloud radio access networks (H-CRANs). They formulated a nonconvex fractional programming optimization issue to manage the optimization of resource allocations. They demonstrated that the performance gains of H-CRANs over the conventional heterogeneous networks and cloud radio access networks are significant. In addition, they proposed an optimal energy-efficient resource allocation solution outperforms the other two baseline algorithms. In [7], the authors discussed the developing requirement for energy efficiency in the next generation networks and the significance of choosing the appropriate energy efficiency metric. Further, they have provides the different methods which can be utilized in the future for optimizing the power of the network. Moreover, they described a system model for energy efficiency improvement with the use of relay selection along with a comparison of various algorithms used for energy efficiency in relay based situations. They have also proposed an energy efficient architecture for $5 \mathrm{G}$ networks with the aim of pursuing green communication for improving energy efficiency of wireless network.

Distributed mobile cloud (DMC) is one of the new schemes for reducing the energy consumption of terminals, where the mobile terminals (MTs) will collaborate using short range (SR) links [8]. In DMC, MTs can share the data assembled from an access point (AP) with one another keeping in mind the end goal being to save energy by staying away from the use of energy-wise unreasonable long range (LR) communications link with the AP. The idea is to use the quick and low-energy SR communications link inside of the cloud rather than using the moderately slow and possibly high power and lossy LR link. In [9], authors have explored the problem of resource allocation and user scheduling for OFDMA networks with DMC, and they have proposed an algorithm that can conspicuously obtain energy efficiency performance. The joint optimization problem was solved by addressing two subproblems, including opportunistic determination of the receiver, subchannel and power allocations with the aim of energy consumption minimization. The authors of [10] propose a theoretical analysis on the energy consumption of the MTs within the cloud. In addition, user scheduling plans were introduced to investigate users participate in receiving from the BS to enhance the energy efficiency. Finally, the authors shows the energy saving beneficial of a mobile cloud and also represented the advantages of supporting wireless power transfer for the mobile cloud. 


\section{Contributions}

In [8], the authors have discussed the possible energy efficiency gains accomplished by DMC, assessed the maximum amount of distance between the SR links, and compared the energy efficiency among the inter-device distance by a number of collaborating MTs. The authors of [11] devoted their work to the power saving schemes for wireless distributed computing networks. However, these commitments concentrated more on the subject of power saving execution of processing instead of that of communications. In [12], SR collaboration among MTs was proposed as a key to diminishing the transmit energy consumption for the transmission from MTs to AP.

The major contribution of this paper is to find the optimal transmit power using computation offloading over DMC collaboration, and is based on wireless transmission to reduce the energy consumption. In the standard DMC without optimal transmit power, when the data is sent to the MTs from an AP, the power consumption is high and will decrease the energy efficiency of the system. However, in the proposed method, the power consumption with high energy efficiency within the cloud in DMC collaboration with a short sensing time is reduced. The main contribution of our work is three-fold:

- We first model the optimal transmit power of the overall transmission process of the DMC, and discuss the potential to obtain lower energy consumption;

- Then, we focus on the energy saving optimization algorithm when the power dissipation of baseband signal processing and RF transmission power are together considered;

- By using baseband signal processing and optimal transmit power, the amount of energy saving can be increased inside the DMC.

The rest of the paper is organized as follows: In Section 4, the system model with a scenario of the proposed method inside DMC network systems is described. The optimal transmit power is described in Section 5 and the energy saving of the system in Section 6. The Simulation results are presented with discussions in Section 7, and finally, Section 8 concludes the work.

\section{System model and scenario description}

The concept of DMC is shown in Fig. 1. A DMC contains a number of resourceconstrained MTs being able to receive the data individually from the AP that are potentially capable of exchanging the received data between each other. As shown in Fig. 1, the MTs in the DMC access the AP through their LR links, being capable of collaborating and sharing the data with other MTs, even those the MTs that would otherwise be out of the AP range. In this regard, using device-to-device (D2D) communication via the SR links makes it feasible.

Considering the fact that MTs are at a unique distance to the AP, they have an alternate quality radio link to the AP. Therefore, each MT also has a different power requirement for successful communication with the AP. The DMC idea uses this knowledge by permitting a certain MT with the best cloud wireless connection in 


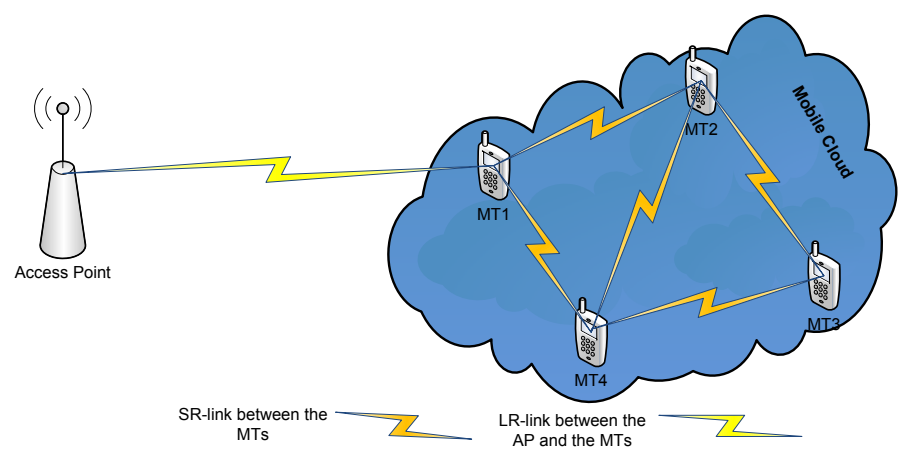

Fig. 1: DMC system model.

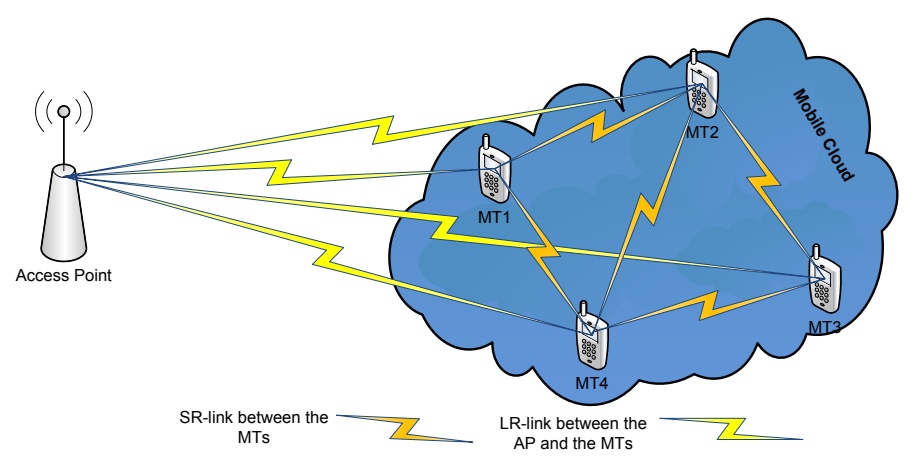

Fig. 2: Round-robin scheduling process.

order to communicate with the AP, and then to spread the data to other MTs in the DMC, subsequently acting as a bridge between the DMC and the AP. The bridge MT is $\mathrm{MT}_{1}$, since in Fig. 1 it is nearest to the AP physically. Thus, it also has the best wireless connection to the AP. Using the DMC collaboration, it is capable of using the good wireless channel of $\mathrm{MT}_{1}$ by handling the transmissions to the AP instead of the cloud. 

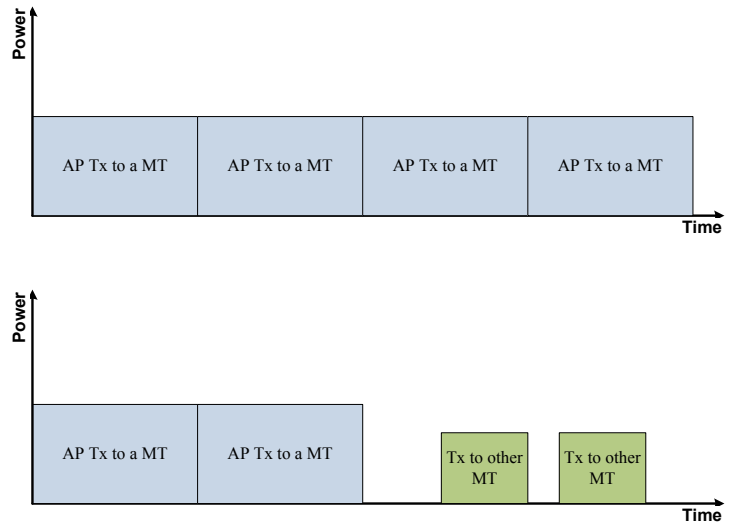

Fig. 3: Comparison of non-collaborative transmit scenario and DMC.

The advantage of this DMC cooperation strategy is reducing the network's general energy and resource consumption, while expanding the data rates for the nodes in the DMC, particularly when contrasted to a more traditional AP to MT link, or even to a more advanced point-to-point communication link. The increased data rates are owing to the better wireless channels, enabling the utilization of a faster connection with more elaborate modulation schemes. As the case presented in Fig. 1 shows, this collaborative scheme is compared to situation in which all of the MTs inside the DMC access the AP in a round-robin (RR) scheduling style, as described in Fig. 2. RR scheduling permits communication to the distant AP as a successive request, even if the nodes with wireless connections from/to the AP are poor. The MTs in the DMC should all be dual-mode devices with two radios. One radio should have an SR wireless capability for inter-device communications, while the other radio should be outfitted with the ability to access the LR broadband for communicating with the AP. In addition, it is essential that all MTs require the same data from the AP in order to broadcast video or digital radio and that all MTs run with an equivalent data rate at their corresponding radios. RR scheduling was chosen for the purpose of consistency as a method to access the AP. Accordingly, the SR connection is significantly faster, maintaining running with a lower power consumption compared to the LR link.

Fig. 3 compares the LR and SR communication standards. The upper part demonstrates the power and time prerequisites for a non-collaborative scenario in which the AP sends data to each MT exclusively with a slower and more energy-hungry LR link, whereas the lower diagram demonstrates the merit of using a DMC when transmitting the same data. In the DMC, the AP needs to send the data to fewer MTs with slower and more energy-hungry LR link. However, in order to enhance the general system energy efficiency, the MTs can then appropriate the received data with each other by using a lower power and a faster SR wireless connection. 


\section{Optimal transmit power consumption}

Transmitter power control components in wireless networks has been used to meet two unique objectives to reduce energy consumption in mobiles, in order to moderate battery life and decrease impedance in the common medium. This further allows more considerable reuse and concurrence of correspondence. A method to overcome the limitations of mobile batteries is to utilize offload computations of the mobile phones in the cloud as a key to reduce energy consumption of the MTs. The idea of offloading computation from mobile phones to wireless processing resources to improve efficiency and reduce energy consumption has been common for more than a decade [13]. In recent years, this idea has received more consideration owing to the huge rise in the complexity of mobile applications, the accessibility of effective clouds and the improved network choices for mobile devices.

In this section, the first criterion we analyze is the reduction of power consumption using offload computations to guarantee the energy saving where the MTs needs to send all necessary data within the cloud to each other from AP involving a free variable $\beta$ and we suppose that the computations have been used in temperature of $T_{0}$. The other system parameters are: $B$ is the bandwidth of the link, $L$ is the number of bits of the data packet, $P_{t}$ is the power consumed to transmit the data from the AP to MTs, and $\lambda_{t}$ is the packet transmission rate to run the MTs applications. Assuming adaptive modulation, the necessary parameter $\beta$ to send the $L$ data packet by $\lambda_{t}$ across a channel of bandwidth $B$ is pertinent to $P_{t}$ as below [14],

$$
\beta=\frac{\lambda_{t} L}{B \log \left(1+\alpha P_{t}\right)}
$$

where

$$
\alpha=\frac{G_{t} G_{r} \lambda^{2}}{(4 \pi)^{2}}
$$

and $G_{t}$ and $G_{r}$ are the transmitter and receiver omnidirectional antenna gains. $\lambda=$ $c / f_{c}$ is the signal wavelength, where $c$ is the speed of light and $f_{c}$ is the carrier frequency.

Using the relations between the $P_{t}$ and $\beta$, the objective performed will be mentioned in terms of the only variable $\beta$ to minimize the transmit power and the improvement issue can be formulated as [14]:

$$
\begin{gathered}
\min _{\beta} \min \left[\beta P_{t}\right] \\
\min _{\beta} \min \left[\frac{\beta}{\alpha}\left(2^{\frac{\lambda_{t} L}{B \beta}}-1\right)\right]
\end{gathered}
$$

subject to (s.t.)

$$
\frac{\lambda_{t} L}{B \log \left(1+\alpha P_{t}\right)} \leq \beta \leq T_{0}
$$


After some forward manipulations between (1) and (5), and solving the quadratic equation for $P_{t}^{*}$ instead of $P_{t}$, the optimal transmit power can be mentioned in closed form as:

$$
P_{t}^{*}=\frac{2^{\frac{\lambda_{t} L}{B T_{0}}}-1}{\alpha}
$$

Transmitted power is needed to calculate the energy of the wireless systems within the cloud as one of the major factors of these systems to contribute to the energy consumption reduction. In this section, the optimal transmit power obtained from (6) has been proposed to increase the energy saving received by this optimal transmit power from the transmitter and receiver omnidirectional antenna gains, working temperature and other parameters used in this section. In addition, Alg. 1 presents the function of optimal transmit power computation.

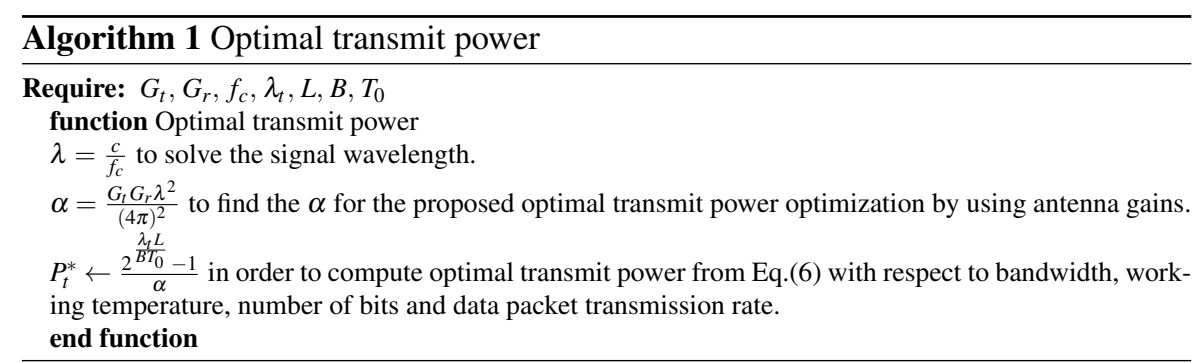

\section{Energy saving}

The total required energy consumption of the network to transfer data to each MT in the DMC collaboration in downlink can be modeled as follows:

$$
E_{\text {total-best-MT }}=A P_{t x}+N\left(M T_{D M C_{t x}}+P_{E}\right),
$$

where $N$ is the total amount of MTs in the DMC, $A P_{t x}$ is the power consumed by the AP when transmitting the data to a MT, and $M T_{D M C_{t x}}$ is the power consumed by the MT when transmitting the data to the DMC. The power dissipation for baseband signal processing can be formulated as [15]:

$$
P_{E}=\left(C_{E}+C_{R} \frac{R_{s, \max }}{R_{s}}\right) R_{s},
$$

where $R_{S}$ is the symbol rate, $R_{s, \max }$ is the maximum symbol rate, and $C_{E}$ and $C_{R}$ are related to system voltage level.

For the RR scheduling conditions, it can be accepted that the wireless link features of the individual DMC MTs vary extensively when imparting to the AP due to the distinctive distances, and, consequently, the radio connection qualities between the MTs and the AP. Owing to this difference, we can present an arbitrary variable 
called channel quality index (CQI) into the aggregate power necessities of the $A P_{t x}$ and $M T_{D M C_{t x}}$, to simulate the emerging irregular quality changes in the radio connection, and to transmit the information effectively. The new variable may be certainly referred to as $A P_{C Q I}$. The aggregate required system energy consumption to download the same measure of data to each MT in the DMC with RR scheduling can be provided as follows:

$$
E_{\text {total }-R R}=N\left(A P_{t x} \cdot A P_{C Q I}\right)
$$

To have the capacity to evaluate the contrast between these strategies, and in this manner, the energy efficiency advantage from the main MT to AP over a RR scheduling, the energy saving can be expressed as follows:

$$
E_{s}=\frac{E_{\text {total }-R R}}{E_{\text {total }- \text { best }-M T}}
$$

The free space simplified path loss model (SPLM) is a general and basic model to examine the path loss in various situations represented as follows [16]:

$$
P_{r}=P_{t}^{*} K\left(\frac{d_{0}}{d}\right)^{\eta}
$$

In this approximation, $\eta$ is the path loss exponent as an empirically or analytically solved component of the carrier frequency. $K$ is a unitless constant relying on the antenna characteristics and average channel attenuation, $P_{r}$ is the received power at the receiver, $P_{t}^{*}$ is the optimal transmitter power, $d_{0}$ is the reference distance assumed to be indoors, and $d$ is the receiver distance.

Since the simplified model is utilized to approach empirical measurements, in some cases, the value of $K<1$ is set to free space path gain at distance $d_{0}$ expecting omnidirectional antennas [17]:

$$
K=10^{\log \left(\frac{\lambda}{4 \pi d_{0}}\right)}
$$

The SPLM raised in (11), in fact, does not calculate the transmission losses themselves, but rather it provides the amount of the RF power received at a specific distance. Accordingly, the SPLM equation was transmuted as follows with a specific ultimate goal to calculate the difference between transmitted power and received power, and develop the actual losses.

$$
P_{l}=P_{t}^{*}-P_{r}
$$

The energy saving calculation from (10) is conducted by isolating the summed expended energies utilized by the AP when communicating in a RR design into the MT of the cloud with the energy consumed in a correspondingly measured DMC collaboration effort case, where the calculation first accounts the closest MT to the AP and sums that calculation to the $N-1$ sized cloud of MTs with a distance of 10 meters to each other in this scenario owing to its simplicity. This could be reformed as:

$$
E_{s}=\frac{N\left(P_{l}\right)}{\left(P_{l}+\left(N-1\left(P_{l}+P_{E}\right)\right)\right)}
$$


Table 1: Simulation parameters

\begin{tabular}{lll}
\hline No. & Parameters & Values \\
\hline 1 & The Size of the DMC & 50 \\
2 & Number of averaging iterations & 200 \\
3 & Average distance between the MTs in the DMC $(d)$ & 5 \\
4 & Temperature in Kelvin $\left(T_{0}\right)$ & $300 \mathrm{~K}$ \\
5 & Reference distance $\left(d_{0}\right)$ & $1 \mathrm{~m}$ \\
6 & Carrier frequency $\left(f_{c}\right)$ & $2 \mathrm{GHz}$ \\
7 & Packet size $(L)$ & $10^{3}(\mathrm{~b} / \mathrm{pck})$ \\
8 & Bandwidth $(B)$ & $2 \mathrm{MHz}$ \\
9 & Speed of light $(c)$ & $3 \times 10^{8}(\mathrm{~m} / \mathrm{s})$ \\
\hline
\end{tabular}

Since energy and power consumption of wireless networks are highly important, a proposed scheme is provided in Alg. 2. As mentioned, the optimal transmit power in (6) has been considered to decrease power transmission by using antenna gains. In this algorithm, for the DMC vector size, the number of MTs will be added to the DMC size vector increasing by every 5 vectors in a loop. Then, the SPLM in (13) appears in the second loop, and after that, the energy saving in (14) is presented in the last loop through iterations.

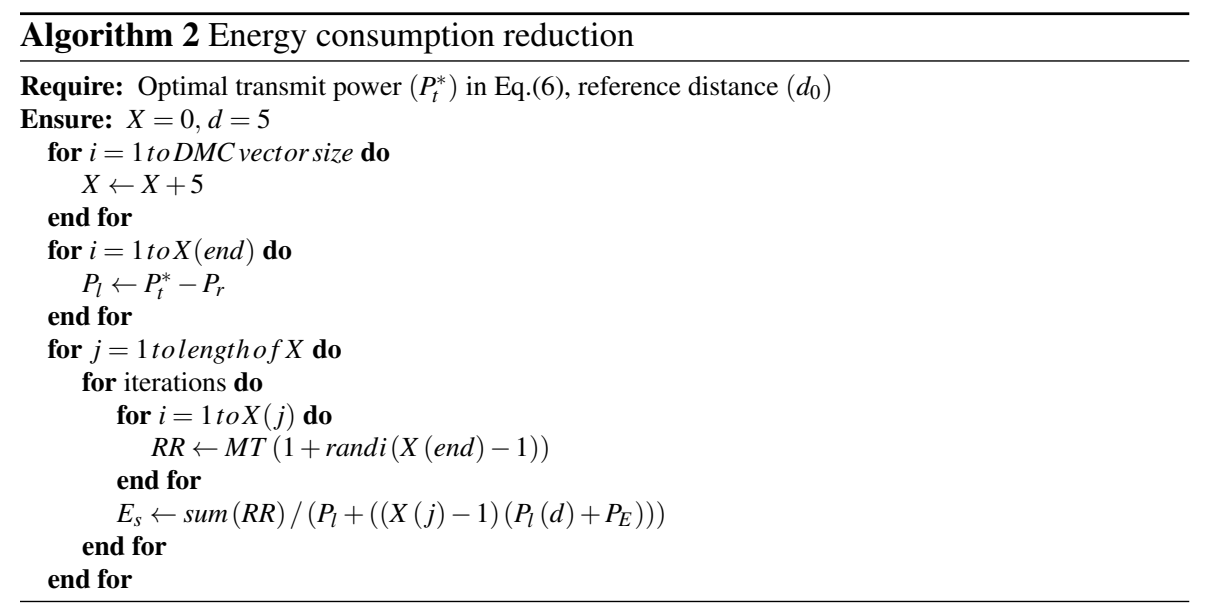

\section{Simulation results}

This section presents the simulation results along with discussions with respect to examining the utilization of the DMC for power consumption reduction, through Monte-Carlo simulations, for the proposed method on a MATLAB environment. The simulation parameters values are according to [8], [18], and summarized in Table 1. To run the simulations, we first obtain the proposed optimal transmit power from (6) 


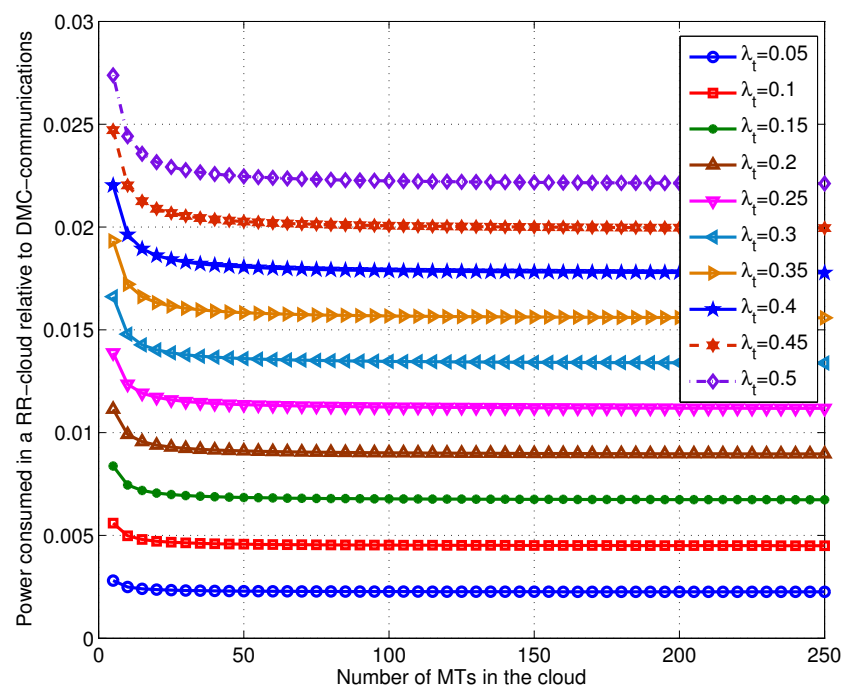

Fig. 4: DMC Power consumption illustration for various packet transmission rates.

to reduce energy consumption. Second, a loop is defined filling the MT vector with the SPLM computations for each MT according to the optimal transmit power. Loops filling the RR vector with random MTs can fill a vector with sums of the RR's in order to achieve energy savings. The energy savings are then evaluated with a 2D-matrix with summed values from the first MT and a number of other MTs with a distance of $d$ by adding the power dissipation. Additionally, the slight variance of the MTs selection randomization inside the cloud where the variance is mitigated by averaging the iterations can be omitted. For baseband energy consumption, we fix $R_{s, \max }=1 \mathrm{MHz}$ and $R_{S}=250 \mathrm{MHz}$ as in [8] and also use the same $C_{E}=8 \times 10^{-8}$ and $C_{R}=10^{-7}$ as in [19].

Fig. 4 presents the proposed scheme power consumption for various packet transmission rates in the RR-cloud vs. varying MTs (MTs varying from 0-250). Here, minimum power is consumed when packet transmission rate is 0.05 and max for 0.5 . Obviously, the energy consumption curve in the proposed method is descending where the power consumed is less than $28 \mathrm{~mW}$ or $0.028 \mathrm{~W}$. The communication network load can be reduced by using the minimum existing number of MTs (MT $=5$ ) inside the cloud for various packet transmission rates, enhancing the system efficiency and also decreasing the environment implications in the way of green communications. To implement this method, we assume the omnidirectional antennas at the nodes $\left(G_{t}=G_{r}=1\right)$ and path loss exponent is 2 . In this figure, the packet transmission rate ranges from 0.05 to 0.5 with increments of 0.05 . As Fig. 4 indicates the power consumption curve is shifted upward for larger packet transmission rates. It can be observed that the power consumed for up to 50 MTs is less than that of five MTs inside the cloud for the relevant optimal transmit power rate, decreasing 


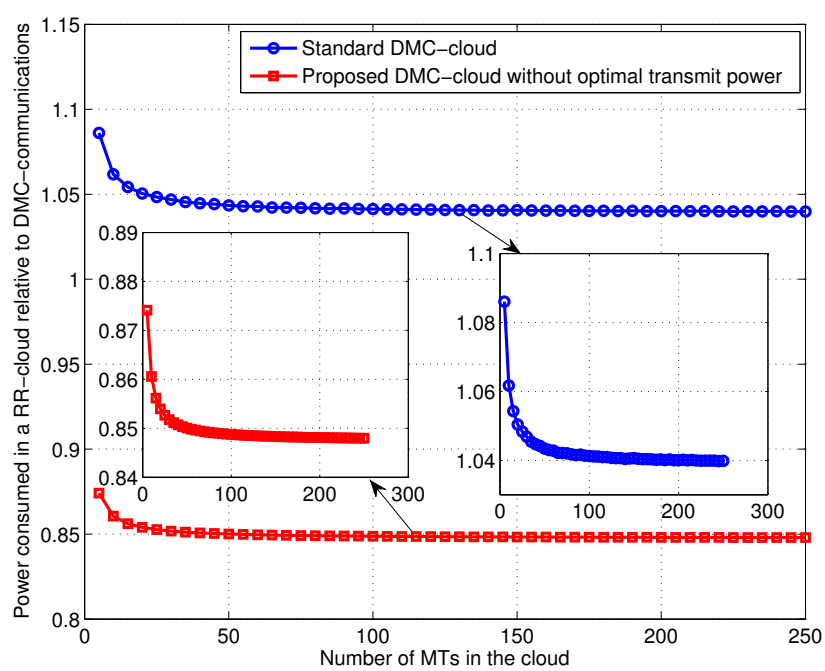

Fig. 5: Comparison of power consumption of our proposed method with the standard DMC inside the cloud for vaious packet transmission rate: Non-optimal transmit power case.

the overall power consumption of the DMC communications through increasing the number of MTs. Furthermore, it is observed from Fig. 4 that the power consumption is increased by increasing the packet transmission rate value, e.g., the power consumption for more than $50 \mathrm{MTs}$ is reduced to lower than $0.023 \mathrm{~W}$ at $\lambda_{t}=0.5(\mathrm{pck} / \mathrm{s})$. Thus, the power consumption reduction of the DMC collaboration communications for various packet transmission rates to have optimum power consumption can be observed.

Figs. 5 and 6 show the power consumption comparison of the DMC scheme with the standard DMC collaboration. From Fig. 5, it is clear that the power consumption of our proposed scheme, without optimal transmit power by using power dissipation, is compared to the standard DMC, without optimal transmit power and without dissipation of power. As shown in Fig. 5, the transmitted power is set to 1, which is an ideal setting for the wireless networks, and obviously, power consumption can be reduced by increasing the number of the MTs in the cloud. In this figure, the power consumption for $250 \mathrm{MTs}$ is $0.848 \mathrm{~W}$ in the red curve. However, the power consumption in the red curve of Fig. 5 for 50 MTs is $0.85 \mathrm{~W}$. From Fig. 6, the proposed scheme is compared to the standard DMC to show the power consumption reduction with respect to optimal transmit power. The increase of energy saving is expected towing to shorter transmit power. As observed in Fig. 6, when $\lambda_{t}>0.05$, the growth of transmit power appears to dominate the increase of $E_{s}$ and energy efficiency begins to decrease. In general, for the same value of $d$, shorter packet transmission rate can obtain higher energy efficiency performance. In Fig. 6, we observe that a DMC with 50 MTs can obtain up to of 1.2 times energy saving that a DMC with 5 MTs. However, 


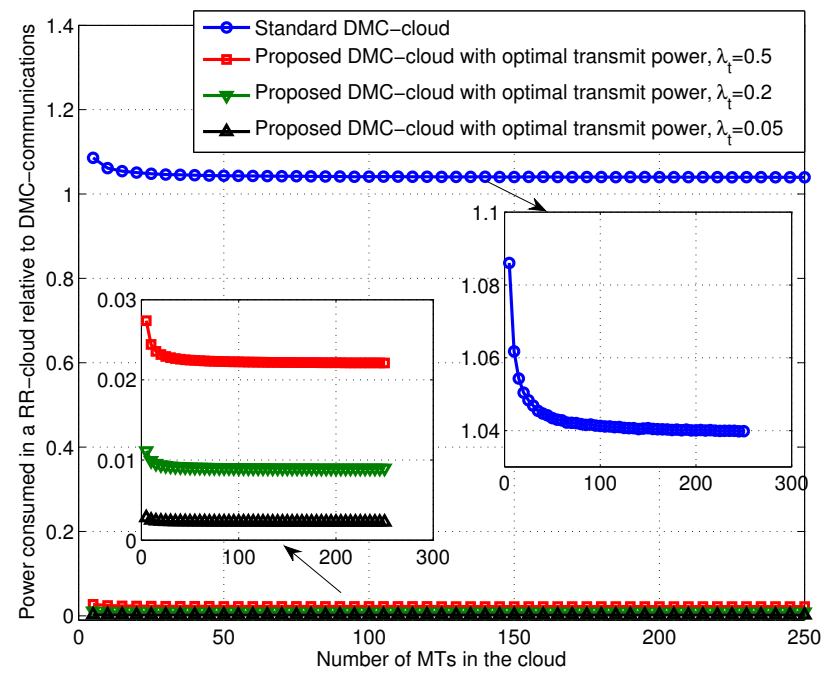

Fig. 6: Comparison of power consumption of our proposed method with the standard DMC inside the cloud for various packet transmission rate: Optimal transmit power case.

the difference of standard and proposed DMC communications is obvious by adding an optimal transmit power when there are more MTs forming a DMC, e.g., a DMC with 50 MTs can achieve a better energy saving performance than that of 10 MTs. Thus, we are able to achieve approximately $80 \%$ of power consumption reduction with optimal transmit power and baseband signal processing.

\section{Conclusion}

Green communications emerge as a research hotspot in order to reduce $\mathrm{CO}_{2}$ emission and energy consumption induced by the wireless networks. In this paper, we have presented an energy efficient approach to reduce the network energy consumption. In particular, the MTs can form a DMC and cooperatively receive from the BS. Therefore, the DMC collaboration is more efficient than direct communication as it requires less power for wireless transmissions than without collaboration. In this work, a closed form analytical expression for optimal transmit power in the DMC is derived aiming at minimizing the system energy consumption. From the simulation study, it can be found that the proposed method can obtain better power saving gain compared with previous approaches.

\section{References}

1. Sunil Vadgama (2009). Trends in Green Wireless Access. FUJITSU Sci. Tech. J., 45(4), pp. 404-408. 
2. Gerhard Fettweis and Ernesto Zimmermann (2008). ICT Energy Consumption-Trends and Challenges. In the 11th International Symposium on Wireless Personal Multimedia Communications (WPMC 2008).

3. Chen Tao, Yang Yang, Honggang Zhang, Haesik Kim, and Kari Horneman (2011). Network energy saving technologies for green wireless access networks. IEEE Wireless Communications, 18(5), pp. 3038.

4. Ferling D., Bohn T., Zeller D., Frenger P., Godor I., Jading Y., and Tomaselli W. (2010). Energy Efficiency Approaches for Radio Nodes. In Future Network and Mobile Summit (pp. 1-9).

5. K. Zhang, Y. Mao, S. Leng, Q. Zhao, L. Li, X. Peng, L. Pan, S. Maharjan, and Y. Zhang (2016). EnergyEfficient Offloading for MobileEdge Computing in 5G Heterogeneous Networks. IEEE Access, 4, pp. 5896-5907.

6. Mugen Peng, Kecheng Zhang, Jiamo Jiang, Jiaheng Wang, and Wenbo Wang (2015). Energy-Efficient Resource Assignment and Power Allocation in Heterogeneous Cloud Radio Access Networks. IEEE Transactions on Vehicular Technology 64(11)

7. Akshita Abrol and Rakesh Kumar Jha (2016). Power Optimization in 5G Networks: A Step Towards GrEEn Communication. IEEE Access, 4, pp. 1355-1374.

8. Zheng Chang and Tapani Ristaniemi (2013). Energy Efficiency of Collaborative OFDMA Mobile Clusters. In IEEE Consumer Communications and Networking Conference (pp. 74-78).

9. Zheng Chang, Jie Gong, Tapani Ristaniemi, and Zhisheng Niu (2016). Energy Efficient Resource Allocation and User Scheduling for Collaborative Mobile Clouds with Hybrid Receivers. IEEE Transactions on Vehicular Technology, 65(12), pp. 9834-9846.

10. Zheng Chang, J. Gong, Y. Li, Z. Zhou, T. Ristaniemi, G. Shi, Z. Han and Z. Niu (2016). Energy Efficient Resource Allocation for Wireless Power Transfer Enabled Collaborative Mobile Clouds. IEEE Journal on Selected Area in Communications, 34(12), pp. 3438-3450.

11. Datla D., Xuetao Chen, Newman T.R., Reed J.H., and Bose T. (2009). Power Efficiency in Wireless Network Distributed Computing. IEEE VTC Spring (pp. 1-5).

12. A. Radwan and J. Rodriguez (2012). Energy Saving in Multi-standard Mobile Terminals through Short-range Cooperation. EURASIP Jour. Wirel. Comm. and Netw. doi:10.1186/1687-1499-2012-159.

13. Karthik Kumar, Jibang Liu, Yung-Hsiang Lu, and Bharat Bhargava (2012). A Survey of Computation Offloading for Mobile Systems. Mobile Netw. Appl., 18(1), pp. 129-140.

14. Barbarossa S., Sardellitti S., and Di Lorenzo P. (2014). Distributed mobile cloud computing over 5G heterogeneous networks. IEEE Signal Processing Magazine, 31(6), pp. 45-55.

15. C. Schurgers (2001). Modulation scaling for Energy Aware Communication Systems. In Proceedings of ISLPED.

16. T.S. Rappaport (1996). Wireless Communications Principles and Practice. N.J.: Prentice-Hall.

17. Andrea Goldsmith (2005). Wireless Communications. Cambridge University Press.

18. Sooksan Panichpapiboon, Gianluigi Ferrari, and Ozan K. Tonguz (2006). Optimal Transmit Power in Wireless Sensor Networks. IEEE Transactions on Mobile Computing, 5(10), pp. 1432-1447.

19. C. Schurgers, O. Aberthorne, and M. Srivastava (2011). Modulation scaling for Energy Aware Communication Systems. In International symposium on Low power electronics and design (pp. 96-99).

20. Zheng Chang and Tapani Ristaniemi (2012). Reducing Energy Consumption via Cooperative OFDMA Mobile Clusters. In IEEE 17th International Workshop on Computer Aided Modeling and Design of Communication Links and Networks (CAMAD) (pp. 162-164).

21. Jayant Baliga, Robert Ayre, Kerry Hinton, and Rodney S. Tucker (2011). Energy Consumption in Wired and Wireless Access Networks. IEEE Communications Magazine, 49(6), pp. 70-77.

22. Wayne Stark, Hua Wang, Andrew Worthen, and Stephane Lafortune (2002). Low-Energy Wireless Communication Network Design. IEEE Wireless Communications, 9(4), pp. 60-72.

23. V. Rodoplu and T. Meng (1999). Minimum Energy Mobile Wireless Networks. IEEE J. Selected Areas in Comm., 17(8), 1333-1334.

24. Karthik Kumar and Yung-Hsiang Lu (2010). Cloud Computing for Mobile Users: Can Offloading Computation Save Energy?. IEEE Computer Society, 43(4), pp. 51-56.

25. Dinesh Datla, Xuetao Chen, Thomas Tsou, Sahana Raghunandan, S. M. Shajedul Hasan, Jeffrey H. Reed, Carl B. Dietrich, and Tamal Bose (2012). Wireless distributed computing: a survey of research challenges. IEEE Communications Magazine, 50(1), pp. 144-152.

26. Adnan Aijaz, Hamid Aghvami, and Mojdeh Amani (2013). A survey on mobile data offloading: technical and business perspectives. IEEE Wireless Communications, 20(2), pp. 104-112. 

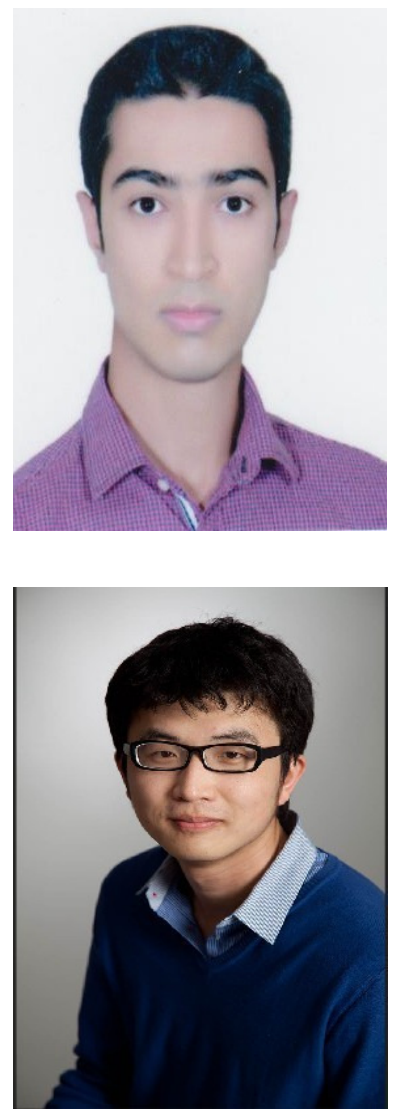

Arash Ostovar was born in Shiraz, Iran, August 4, 1991. He received his B.Eng. degree in Information and Communications Technology (ICT) Engineering from Shiraz Pasargad Higher Education Institute, Shiraz, Iran, in 2013, and the M.Sc. degree in Telecommunications System Engineering from Shiraz University of Technology, Shiraz, Iran, in 2015. Currently, he is a Ph.D. applicant in Electrical Engineering (Telecommunications System) and his current research interest lies in the field of wireless communications, signal processing, cognitive radio networks, optical wireless communications, internet of things, and green communications.

Zheng Chang received the B.Eng. degree from Jilin University, Changchun, China in 2007, M.Sc. (Tech.) degree from Helsinki University of Technology (Now Aalto University), Espoo, Finland in 2009 and Ph.D. degree from the University of Jyväskylä, Jyväskylä, Finland in 2013. Since 2008, he has held various research positions at Helsinki University of Technology, University of Jyväskylä and Magister Solutions Ltd in Finland. He was an visiting researcher at Tsinghua University, China, from June to August in 2013, and at University of Houston, TX, from April to May 2015. He has been awarded by the Ulla Tuominen Foundation, the Nokia Foundation and the Riitta and Jorma J. Takanen Foundation for his research work. Currently he is working with University of Jyväskylä and his research interests include signal processing, radio resource allocation, cross-layer optimizations for wireless networks, and green communications. 